\title{
Development of a 6 Degree of Freedom Robotic Micromanipulator for Use in 3D MEMS Microassembly
}

\author{
Nikolai Dechev ${ }^{1}$, Lu Ren ${ }^{2}$, William Liu ${ }^{2}$, William L. Cleghorn ${ }^{2}$, James K. Mills ${ }^{2}$ \\ ${ }^{1}$ Department of Mechanical Engineering, \\ University of Victoria, PO Box 3055, Stn. CSC \\ Victoria, British Columbia, Canada, V8W 3P6 \\ ${ }^{2}$ Department of Mechanical and Industrial Engineering \\ University of Toronto, 5 King's College Road \\ Toronto, Ontario, Canada, M5S 3G8
}

dechev@me.uvic.ca ren@mie.utoronto.ca, cleghrn@mie.utoronto.ca,mills@mie.utoronto.ca

\begin{abstract}
This paper describes the design and development of a 6 degree of freedom robotic manipulator used in the assembly of three-dimensional MEMS (micro electromechanical systems) microstructures. The robot employs a highly innovative mechanical design for the rotational axes to provide unprecedented access to a microchip substrate for microassembly operations. The first three axes of the robotic manipulator are orthogonally mounted linear stages providing Cartesian positioning of the chips beneath the end effector (microgripper). A rotational stage (alpha) mounted on the distal end of these three Cartesian axes allows the MEMS chip to be rotated. Two more degrees of freedom (beta and gamma) are serially mounted to the base frame, allowing for two degrees of rotation of the end effector. This configuration permits assembly of micro-parts on the surface of a MEMS chip at any orientation angle to the surface, within the limits of the workspace of the manipulator and the resolution of the motors. The end effector employs a standard tungsten probe with a passive microgripper bonded to it, which is used for grasping micro-parts. A software system has been developed to allow automatic operation of the manipulator. Preliminary assembly tests confirm the usefulness of the proposed design.
\end{abstract}

Index Terms - Micromanipulator, MEMS, Microassembly, 6 DOF, Large Workspace.

\section{INTRODUCTION}

The objective of this work is the design and development of a 6 DOF (degree of freedom) robotic micromanipulator with which MEMS devices can be both manipulated and assembled. Previously, a 5 DOF robotic micromanipulator [1, 2] has been developed for the assembly of MEMS micro-parts. While very useful, the previous system had inherently limited accessibility to the MEMS chips, permitting assembly of parts in approximately orthogonal directions to the MEMS chip surface, i.e. $90^{\circ}$ to the chip surface or parallel to the chip surface. In this work we have developed a highly innovative robotic micromanipulator with unprecedented 6 DOF access to the surface of a MEMS chip allowing for assembly of MEMS micro-parts at essentially any angle relative to the substrate.

The new system, as shown in Fig. 1, is able to simultaneously rotate and translate micro-parts with respect to the MEMS chip in 3 rotational DOF $(\alpha, \beta$ and $\gamma)$ and 3 translational $\operatorname{DOF}(x, y$ and $z$ ). The useable rotational workspace is: $360^{\circ}$ about $\alpha, 180^{\circ}$ about $\beta$, and $110^{\circ}$ about $\gamma$, although, larger rotational ranges are possible for certain configurations of the axes.

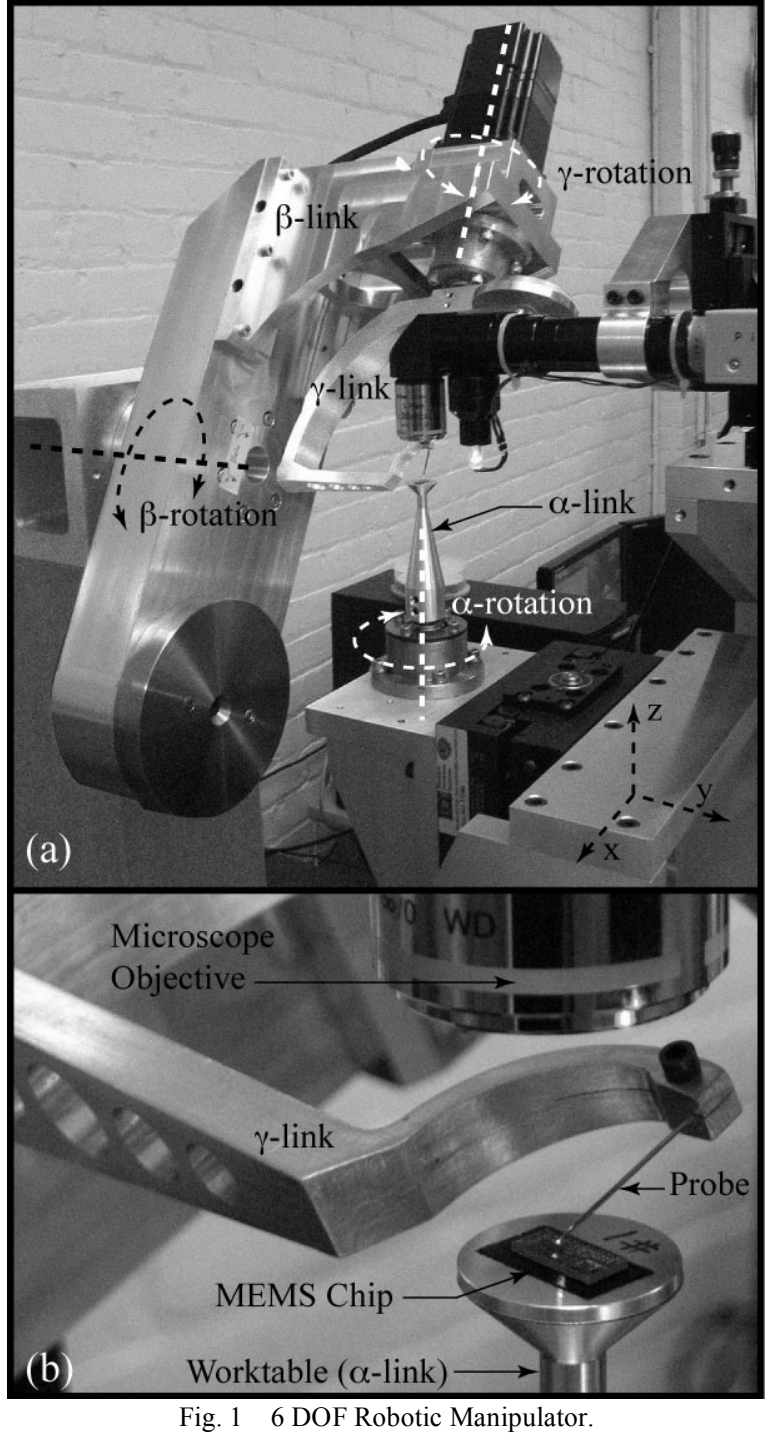

It is noteworthy that this large rotational workspace is achieved while under the full view of a video microscope with 0.8 micron optical resolution. The general aim of this research is to facilitate the integration of a diverse set microcomponents, into more complex devices, by providing a microassembly process. By developing this 6 DOF robotic manipulator, 3D MEMS can be assembled by a sequential process of grasping, manipulating and joining together various 
micro-parts, which range from 60 to 300 microns in overall size. The micro-parts and the microgrippers that are used to grasp them, are fabricated side by side on a silicon chip, fabricated by the MUMPS (Multi-User MEMS Processes) [3] process.

A literature survey of microassembly research, followed by a brief description of the proposed $6 \mathrm{DOF}$ microassembly process, are presented in Section 2 . Section 3 describes the design, methodology and operation of the proposed 6 DOF micromanipulator, and the interface process to attach a MEMS microgripper to the end effector. Section 4 describes the software interface system developed to allow users to program the robot for automatic task execution. Finally, Section 5 provides recently obtained assembly results utilizing this new robotic micromanipulation system.

\section{BACKGROUND}

There are a number of different microassembly approaches used by others including: parallel (batch) microassembly, self-assembly and serial (sequential) microassembly. Parallel microassembly systems assemble microstructures at multiple assembly sites simultaneously. Parallel approaches include 'flip-chip' or 'batch transfer' microassembly [4]. Another parallel approach involves selfassembly systems, whereby the constituent micro-parts will assemble themselves spontaneously while under the influence of an external force such as heat, magnetism or centrifugal force. Some examples of self-assembly systems are solder surface tension self-assembly [5], plastic deformation magnetic assembly (PDMA) [6], and centrifugal force assembly [7]. Serial microassembly is a sequential process, in which assembly tasks are performed one after the other. To complete one assembly, a series of sub-tasks are required, such as grasping micro-parts with a grasping tool, manipulating them, and joining them to other micro-parts. Generally, serial microassembly can be used to create microstructures that are more complex than parallel systems. This is because serial assembly allows for the translation and orientation of individual micro-parts in up to 6 DOF and allows for closed loop control during assembly, as opposed to open-loop assembly of several or more micro-parts in parallel. Other robotic-based serial microassembly systems that make use of robotic manipulators equipped with microgrippers include $[8,9]$ or those equipped with micro-tweezers $[10,11]$.

The proposed robotic micromanipulator performs serial microassembly to sequentially assemble a set of micro-parts. Prior to starting the assembly process, a passive microgripper [2] must be bonded to the end effector probe. Next, each micro-part to be assembled into a microstructure must go through five steps, which are: (1) the micro-part is grasped by the passive microgripper, (2) the micro-part is removed from the chip substrate, (3) the micro-part is translated and rotated through space in up to $6 \mathrm{DOF},(4)$ the micro-part is joined to the target joint site, and (5) the micro-part is released. This process is repeated as necessary until the assembly is complete. A highly detailed description of this process, as developed in past work and applied to this work, is described in [2].
In order to assemble micro-parts together into 3D microstructures, micro-joints are used. The micro-joints used in this work are based on mechanical fastener [12] designs, which provide a number of advantages. Mechanical fasteners require no intermediary material or glue, and they require no additional post-processing of the chip to create a joint. As a result, they can be rapidly made in several seconds or less. Micro-mechanical joints can be designed for high rigidity, and are based only on geometry, which allows for a wide range of micro-part materials to be used in a microstructure.

\section{MANIPUlator DeSIGN AND Methodology}

\section{A. System Requirements}

The new robotic micromanipulator was designed to meet a number of requirements, to increase the general microassembly capability. The major system requirements are presented below, and all have been met or exceeded:

(a) The system must provide $6 \mathrm{DOF}$ for manipulation of micro-parts, so they may be assembled in any possible orientation with respect to other microstructures.

(b) The repeatability of the system must be $+/-2$ microns.

(c) The system must provide a large rotational range for the rotational axes, to maximize the number of different operations that can be performed. It is required to have at least $360^{\circ}$ of rotation about the $\alpha$-axis, $180^{\circ}$ about the $\beta$-axis and $90^{\circ}$ about the $\gamma$-axis. With the appropriate design of a microgripper and a micro-part's 'grasping interface' [2], this range of rotation will allow for assembly of micro-parts at essentially any angle relative to the substrate.

(d) The rotational linkages must have very high stiffness, in order to minimize excitation of the robot from external vibrations, from self-generated vibrations and to minimize gravitational deflection in different configurations.

(e) The system must provide for full and simultaneous utilization of all angles within the specified rotational range, with no self-interference and no 'unusable areas'.

(f) The system will use tele-robotic control or automated vision-based control. Therefore, this requires that the end effector (probe tip with bonded microgripper) be in view of the microscope optics at all times.

(g) The microscope system must provide sub-micron resolution, since the system routinely assembles micro-parts with joint features that are 4 to 12 microns in size, with clearances of 2 to 6 microns for micro-joint formation.

\section{B. Design and Methodology}

The design solution developed to meet these system requirements is based upon a 3 DOF common-rotationalcenter mechanism. To better explain the design methodolgy, we will first describe the 'ideal design' for the system, and its merits. Then, as additional design requirements are incorporated into the design, the 'final design' will be described. Consider the 'ideal design' illustrated in Fig. 2(a). 


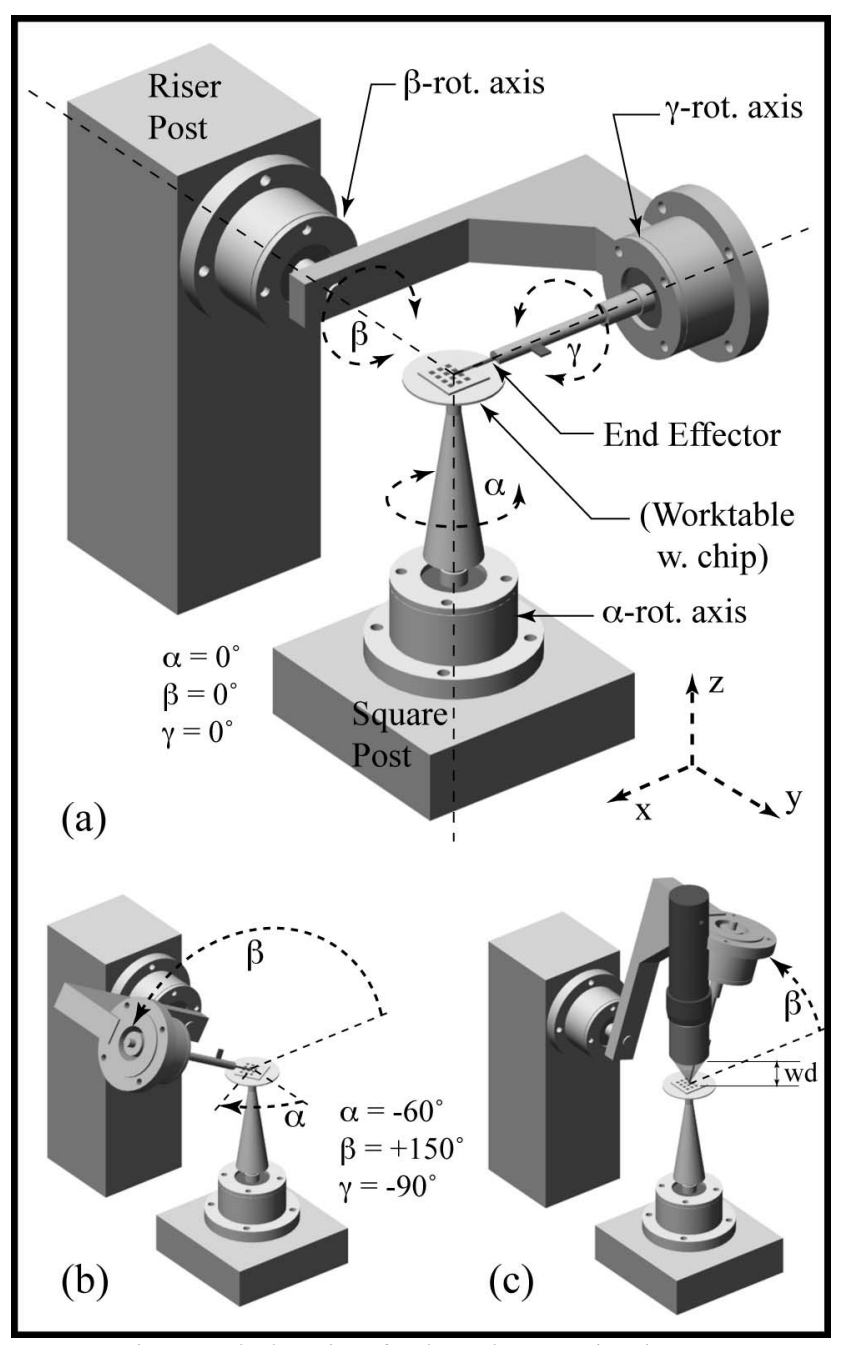

Fig. 2 'Ideal Design' for the Robot Rotational Axes.

The 'ideal design' is one in which the centerlines of each of the three rotational axes pass through a common point in space, regardless of the rotational orientation of any of the three axes. This point in space would coincide with the tip of the end effector, to which a microgripper would be bonded. Fig. 2(a) illustrates how the $\gamma$-rotational axis is mounted onto the $\beta$-rotational axis, which is in turn mounted to a riser post (kinematic ground). The $\beta$ and $\gamma$ rotational axes are configured in such a way that their centerlines will always meet at the common center. The $\alpha$-axis is mounted on the square post, and rotates about a centerline that passes through the common center. The $\alpha$-rotational axis carries the worktable on its top, to which a MEMS chip would be mounted. However, this 'ideal design' must be modified, since $x, y$ and $z$ translation must be provided to the worktable in order to reposition micro-parts. Therefore, assume that the $x, y$ and $z$ axes can reside within the area shown as the square post. During manipulator operation, the $\alpha$-rotational axis will frequently be translated out of alignment with the common center of the $\beta$ and $\gamma$ rotational axes, due to the need to translate to different locations on the MEMS chip. To overcome this problem, the $x$ and $y$ translation axes can be coordinated with the $\alpha$-rotational axis (in software), to create a 'virtual center of rotation' that coincides with the commonrotational-center.

The robotic manipulator was designed to employ the common-rotational-center concept for a number of reasons. (1) The main advantage is that the configuration illustrated in Fig. 2(a) has a large rotational workspace, with $360^{\circ}$ of rotation possible for the $\alpha$-rotational axis, and $360^{\circ}$ for the $\gamma$ rotational axis. The $\beta$-rotational axis also has a large rotational range, but is limited to $180^{\circ}$ due to the use of a flat MEMS chip. (2) Another advantage is that system is inherently non-sensitive to rotational disturbances or small rotational errors. If there are rotational disturbances due to external vibration or small rotational errors in motor position, they remain proportionally small at the micro-scale, since the microgripper tip is at the center of rotation. Conversely, if a non-centered design is used, where the end effector is at some distance from the center of rotation, any rotational disturbance is amplified, with the potential for large end effector deviations. (3) Another advantage is that end effector tip remains approximately at the same location in Cartesian space during 3 DOF rotations. This allows for easy viewing of the end effector with a machine vision microscope and does not require tracking of the end effector with the microscope translation stage. (4) Another advantage to having the end effector tip at approximately the same location in Cartesian space is that it allows for a small $x, y$ and $z$ translation stage to be used to move the worktable. Although this advantage has not been implemented with our system, it is noteworthy. Overall system positional accuracy can be higher, and positional resolution can be higher, when using small travel $x$, $y$ and $z$ translation stages. Some types of small travel translational stages employ piezo-electric actuators and elastically compliant mechanisms, which generally have higher accuracy and higher resolution than traditional long travel, lead-screw-based stages.

\section{Final Design for the 6 DOF Robotic Manipulator}

The final design of the robotic manipulator was developed by integrating the microscope system with the 'ideal design'. This was a challenging task, since placement of a standard microscope would limit the rotational range of the robot about the $\beta$-rotational axis. Consider Fig. 2(b), which shows the manipulator (with no microscope) rotated to a configuration where $\alpha=-60^{\circ}, \beta=150^{\circ}$, and $\gamma=-90^{\circ}$. To view the MEMS chip, a standard microscope must be positioned perpendicularly to the MEMS chip, as shown in Fig. 2(c). However, the microscope tube will interfere with the $\beta$ rotation and therefore limit the rotation to less than $90^{\circ}$. In an attempt to resolve this problem, a search was done to find a microscope system with a large $w d$ (working distance) of 150 $\mathrm{mm}$ or more, where $w d$ is the distance between the objective lens and the MEMS chip. It was hoped that with a large $w d$, the $\gamma$-rotational axis could be designed to pass underneath the lens, while the $\beta$-rotational axis was rotated. However, microscope objectives with such a large $w d$ have an inherently 
low resolution, as defined by the Rayleigh resolution and the definition of NA (numerical aperture). To achieve a desired optical resolution of 0.7 to 0.9 microns, the $w d$ of specialized commercially available objectives was in the range of 15 to 24 $\mathrm{mm}$, which is too small to fit the $\gamma$-rotational axis underneath the lens. This presented a major problem since resolution is critical for the microassembly joint task, which thereby limits $w d$, yet the $\beta$-rotational axis is required rotate by $180^{\circ}$ to allow for full utilization of many different 6 DOF microassembly tasks. To overcome this problem, an innovative design is proposed whereby the $\gamma$-rotational end effector $(\gamma$-link as shown in Fig. 1(a)) passes simultaneously, underneath and overtop of, the microscope objective. This concept is illustrated in Fig. 3. By utilizing this concept, the 'concurrent useable, rotational workspace' of the robotic manipulator is: $0^{\circ}<\alpha<360^{\circ}, 0^{\circ}<\beta<180^{\circ}$, and $-55^{\circ}<\gamma<+55^{\circ}$.

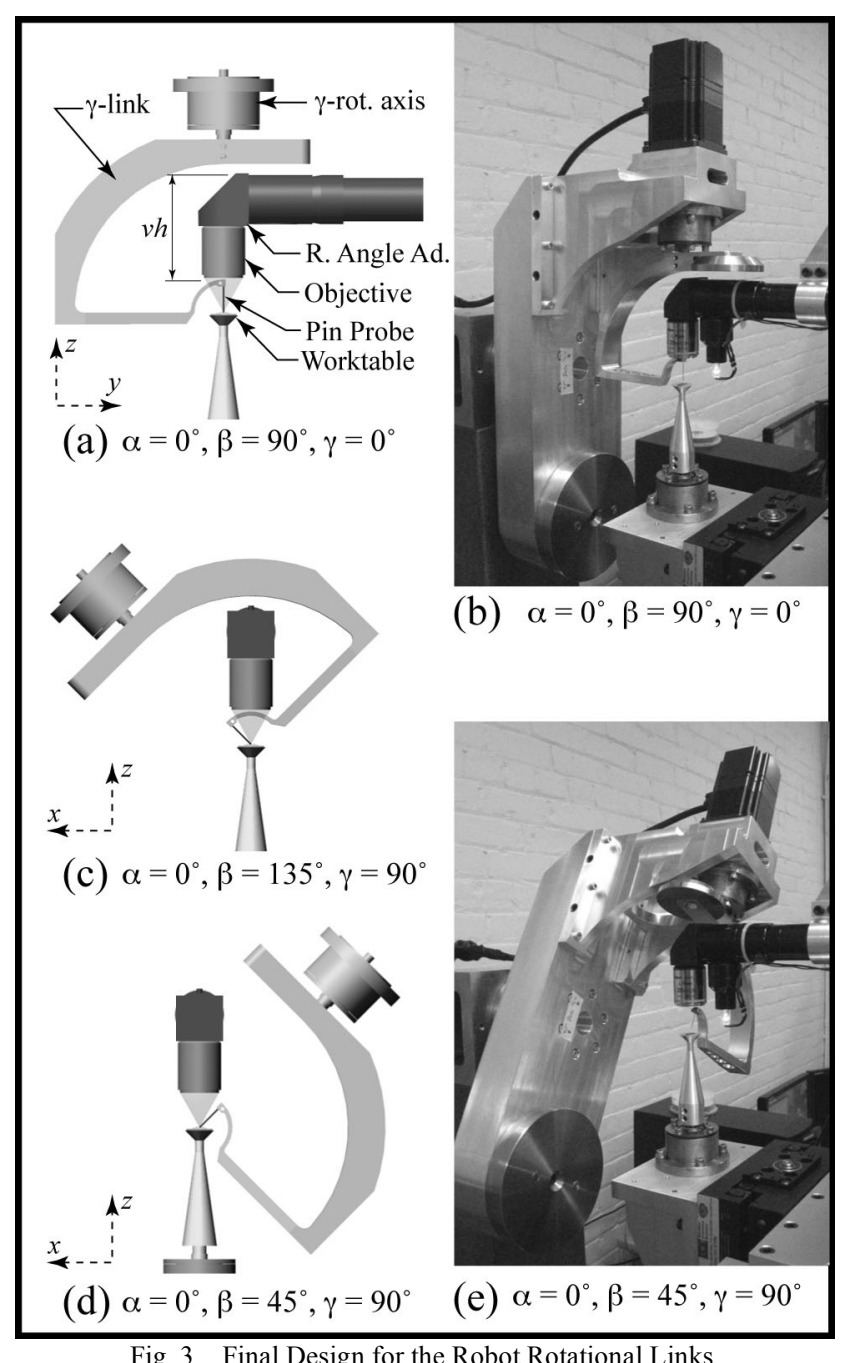

Fig. 3(a) illustrates a side view (y-z plane) of the robotic manipulator, where $\gamma=0^{\circ}$ and $\beta=90^{\circ}$. In order to allow the $\gamma$ link to simultaneously pass over and under the microscope objective, the microscope was designed and constructed with a right angle adapter to bend the microscope tube by $90^{\circ}$. The key to this design was to minimize the vertical height, $v h$, of the objective lens and the right angle adapter. The length of $v h$ affects the overall size of the $\gamma$-link. Therefore, a larger length $v h$ would require a larger $\gamma$-link, which in turn increases the $\beta$-link geometry as well. Large linkages are undesirable since they would have lower stiffness, and are more prone to vibration. To keep $v h$ to a minimum, a Nikon CFI60 L-Plan Epi 20X objective (model MUE 30200) was found to be the best selection. It has a NA of 0.35 (which provides an acceptable resolution), a $w d$ of $24 \mathrm{~mm}$, and a body height of $60 \mathrm{~mm}$. The objective was combined with a right angle adaptor from Infinity Photo Optical, to create an assembly with a $v h$ of $95 \mathrm{~mm}$. The large $w d$ of the objective also allows for sufficient space under the lens for the probe pin and distal portion of the $\gamma$-link. Fig. 3(b) shows a picture of the completed robot in the same configuration as that of Fig. 3(a).

Fig. 3(c) illustrates a front view ( $x-z$ plane) of the robot in a configuration that helps to illustrate how the shape of the $\gamma$ link was developed. In this configuration, the $\gamma$-link is at $\gamma=$ $90^{\circ}$ from its home position (which is outside the concurrent rotational workspace of the robot), and the $\beta$-link is held at $\beta=$ $135^{\circ}$. This configuration allows the $\beta$-rotational axis to rotate in a range from about $30^{\circ}$ to $150^{\circ}$, which defines the inner shape of the $\gamma$-link. Below this range, the $\gamma$-link will collide with the worktable, and above this range, the $\gamma$-link will collide with the microscope objective. Fig. 3(d) illustrates the same view and same $\gamma$-link orientation, but with $\beta=45^{\circ}$. In this case, note how the worktable defines the shape of the distal portion of the $\gamma$-link. Fig. 3(e) shows a picture of the robot in the same configuration as that of Fig. 3(d).

\begin{tabular}{|ccc|}
\hline $\begin{array}{c}\text { Beta Axis } \\
\text { Position (deg) }\end{array}$ & $\begin{array}{c}\text { Gamma Axis } \\
\text { Range of Rot. (deg) }\end{array}$ & $\begin{array}{c}\text { Microscope View of } \\
\text { Target Chip (deg of } \gamma \text { ) }\end{array}$ \\
10 & 66 to $-55 *$ & Clear View \\
20 & 67 to -56 & Clear View \\
25 & 77 to -61 & Clear from 77 to -58 \\
30 & 175 to -66 & Clear from 175 to -56 \\
40 & 174 to -109 & Clear from 174 to -55 \\
50 & 171 to -115 & Clear from 171 to -50 \\
60 & 168 to -125 & Clear from 168 to -43 \\
70 & 164 to -137 & Clear from 164 to -28 \\
80 & 160 to -141 & Partially Clear \\
90 & 156 to -145 & Partially Clear \\
100 & 151 to -150 & Partially Obstructed \\
110 & 146 to -155 & Partially Clear \\
120 & 141 to -160 & Partially Clear \\
130 & 137 to -164 & Clear from 27 to -164 \\
140 & 127 to -168 & Clear from 43 to -168 \\
150 & 115 to -171 & Clear from 50 to -171 \\
155 & 109 to -174 & Clear from 55 to -174 \\
160 & 67 to -175 & Clear from 56 to -175 \\
170 & 61 to -77 & Clear from 58 to -77 \\
$180 *$ & 56 to -67 & Clear View \\
\hline
\end{tabular}

Table 1 Rotational Range of $\gamma$-axis vs. $\beta$-axis Orientation. *Note: due to $10^{\circ}$ taper of probe tip, probe tip cannot touch substrate when $\beta<5^{\circ}$ or $\beta>175^{\circ}$. 
The results of the robotic manipulator for the 6 DOF manipulation of micro-parts are very good. Table 1 lists the rotational workspace of the robot, showing the range of rotation of the $\gamma$-axis, with respect to the orientation of the $\beta$ axis in $10^{\circ}$ increments. Outside of these ranges, the $\gamma$-link may collide with either the microscope, the objective, the $\alpha$-link or the $\alpha$-axis. Values between increments can be approximated by interpolation. Also, a singularity exists when $\beta=90^{\circ}$ (as shown in Fig. 3(a-b)), since the $\alpha$-axis and $\gamma$-axis are co-axial at this point. Note that the rotational workspace in the table is much larger than the stated 'concurrent rotational workspace'. For example, for $\beta$-axis orientations between $30^{\circ}$ to $150^{\circ}$, the $\gamma$-axis has a range of $-109^{\circ}$ to $+109^{\circ}$, and this range increases as the $\beta$-axis range decreases. Also note that the microscope view is partially obscured for certain configurations, such as the one illustrated in Fig. 3(c). It can be seen that the distal portion of the $\gamma$-link lies between the objective lens and the MEMS chip. Surprisingly, the view of the MEMS chip is not blocked at all, but appears slightly dimmer in the region that is covered by the $\gamma$-link. This is because only a portion of the lens is covered, which allows the image to be formed by the remaining light entering the objective lens. The third column in Table 1 summarizes the clarity-of-view of the MEMS chip, and those ranges of rotation that are partially clear (slightly dimmer). When $\beta=90^{\circ}$, the view is very dim and slightly defocused, due to the large amount of obstructed view. With a smaller and thinner design for the distal end of the $\gamma$-link, the dimming effect can be even further reduced.

\section{Video Microscope System}

The video microscope system is the primary source of feedback during tele-robotic operations or automated machine-vision operations. As described in the previous section, the system is based upon the use of a Nikon objective lens, connected to a $90^{\circ}$ right angle adapter from Infinity Photo Optical. The objective lens and microscope tubes are infinity corrected, allowing for greater flexibility in designing the optical system. A co-axial illuminator is connected in-line with the optics to provide illumination of the MEMS chip. A non-traditional light source is used, which is a high brightness LED (light emitting diode) with 5-10 candelas of output. LED light has been extensively used in this work, and offers a number of advantages [13]. The primary advantage is that LED light is fairly monochromatic. This is important since the optical resolution of a microscope system is directly related to the wavelength used, as per the Rayleigh resolution formula (resolution $=0.61 \times$ wavelength $/ \mathrm{NA}$ ). As such, we use blue $(450 \mathrm{~nm})$, green $(520 \mathrm{~nm})$ and red $(660 \mathrm{~nm})$ LEDs for various purposes. Using blue light, the optical resolution of this system is 0.8 microns. The video camera used is a digital, monochrome unit from Pixelink (model \# A741) with a 2/3" $\mathrm{CCD}$ and a resolution of $1280 \times 1024$ pixels. Combined with the microscope system, the resulting field of view is $427 \mathrm{H} \times$ $320 \mathrm{~V}$ microns, and the depth of focus is 1.5 microns. This provides for a digital resolution of $\approx 0.33 \mathrm{microns} / \mathrm{pixel}$ as visible on a monitor (not to be confused with the 'true' optical resolution of 0.8 microns). The camera is connected via a Firewire interface directly to the $\mathrm{PC}$, and requires no capture card, as all information is handled by software. This system allows for adjustment of several parameters including contrast, brightness, shutter and gain, and allows for capture of still images and movies.

\section{E. Robot Structural Design}

The design details of the robotic manipulator are presented. Extensive use of CAD (computer aided design) was made during the design of the system. All components were modelled in 3D and a 'virtual model' was assembled using Visual Nastran software to assist with the design of the kinematics and dynamics of the system. Extensive FEA (finite element analysis) and re-design was carried out during the design of the rotational linkages to maximize the stiffness and keep the first mode of vibration of individual linkages above $250 \mathrm{~Hz}$. Stepper motors from Oriental Motor (model \# AS66MA for the $\alpha$ and $\gamma$ axes, and model \# AS911AA for the $\beta$-axis) with up to $10,000 \mathrm{steps} / \mathrm{rev}$, were selected to drive the three rotational axes of the robot since they naturally exhibit very steady positioning in the 'hold' state. This is important for microassembly tasks, since the rotational axes are usually in a 'hold' state, while the translational axes are moved along a trajectory during micro-joint insertion/formation tasks. Counterweights are used to neutrally balance the $\beta$-axis and $\gamma$ axis to minimize the holding torque requirements for the motors. The three rotational axes have been carefully designed to maximize the repeatability of the robot. This is done by designing each rotational axis as 'direct drive' (no gearbox), where the axis shaft is directly coupled to the motor shaft. Each axis shaft is supported using duplex, preloaded, high precision, spindle bearings to achieve axial and radial runouts of less than 1.3 microns and zero-backlash. The use of these bearings also allows for extremely stiff joints, along the remaining constrained degrees of freedom of a rotational joint. The $x, y$ and $z$ axes are comprised of Danaher Precision Systems Ltd. crossed-roller bearing stages, driven by ballbearing lead screws with a $2 \mathrm{~mm}$ lead. Combined with the stepper motors, this allows for motion resolution as low as 0.2 microns. Lastly, the microscope system is mounted on an independent 3-axis translation stage. This allows the microscope to be moved independently of the robot axes, for various calibration and set-up purposes.

\section{F. Microgripper Bonding}

A microgripper must be bonded to the tip of the end effector probe, before any microassembly operations can be performed. All microgrippers used in this work are fabricated alongside the micro-parts on the same MEMS chip [2]. The bonding process consists of seven tasks, which are: (1) Locate the microgripper to be bonded, with respect to the probe tip and record the location, as shown in Fig. 1(b). (2) Lower the worktable that carries the MEMS chip, away from the probe to 
provide clearance to apply an adhesive to the probe tip. (3) Apply a UV (ultra-violet) curable adhesive to the probe tip.

(4) Re-position the worktable under the probe tip to the previously recorded location at the desired microgripper. (5) Allow the adhesive to flow from the probe tip onto the microgripper bonding pad for a few seconds. (6) Radiate the probe tip with UV light for 30 seconds, to cure the adhesive. (7) Move the MEMS chip down and away from the probe tip, thereby detaching the microgripper from the MEMS chip. The microgripper is now ready to be used for grasping tasks. Fig. 4 shows an SEM (scanning electron microscope) image of a microgripper bonded to the tungsten probe tip.

The microgripper shown was bonded to the probe tip while the probe was held at $20^{\circ}$ to the chip surface $\left(\beta=20^{\circ}\right)$. Normally it would be desirable to bond the microgripper such that there is a minimum angle between its plane and the centerline axis of the probe. Such a configuration would maximize the useable assembly orientations of the microgripper. However, most probe tips have a finite taper angle of usually $10^{\circ}$, therefore, the minimum possible bonding angle for the probe would be half this value (i.e. $\beta=5^{\circ}$ ). It is important to note that the probe can be positioned at 'any desired orientation' about the $\alpha$-axis, $\beta$-axis and $\gamma$-axis, prior to being bonded to a microgripper. There may be different reasons for choosing non-standard gripper-to-probe orientations, such as the need for the microgripper to perform assembly operations at unusual angles.

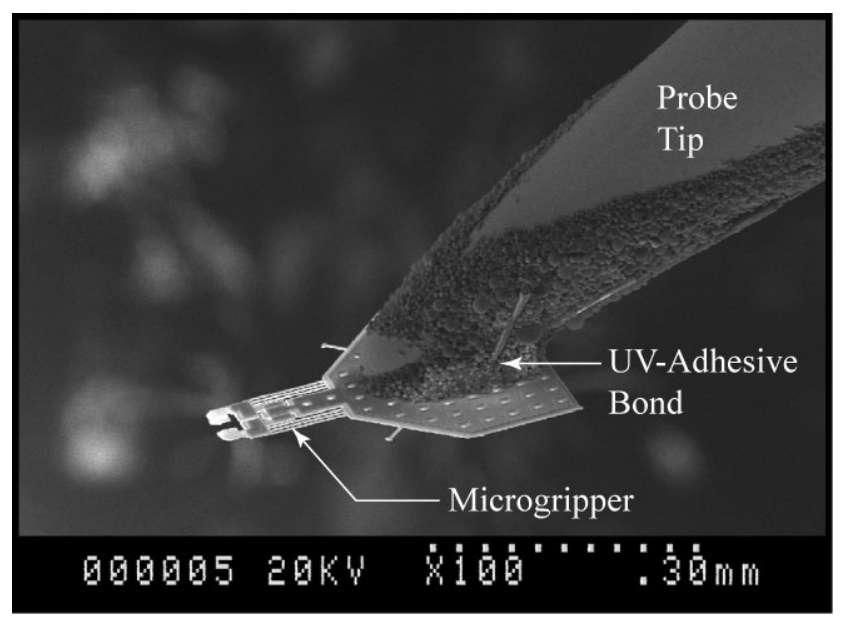

Fig. 4 SEM Image of Microgripper Bonded to Probe.

The methodology of using microgrippers as end effectors with the robotic manipulator is worth noting. There are usually a dozen or so microgrippers fabricated alongside the micro-parts on the same chip. Of these, there may be a few different designs corresponding to different grasping or assembly needs [14] for different styles of micro-parts. In this sense, the microgrippers of this work can be considered as 'disposable tools' that are bonded to the robot, used as needed, and then discarded. The same microgripper may be used for hundreds of operations or for only one operation, before it is changed with an alternate design for another type of procedure. For this reason, it is important for the bonding system to be reversible, fast, and reliable. As such, the UV adhesive system was adopted, since the bond is rapid and the adhesive can be dissolved rapidly when changes are required. The preliminary results of the bonding system are good. Thus far, 20 microgrippers have been successfully bonded to the robot out of a total of 23 attempts ( $87 \%$ success). Presently, the time required to manually remove a microgripper and bond a new microgripper is 10 minutes, although this could be reduced to about 2 minutes if the process is automated.

\section{SOFTwARE INTERFACE FOR AUtOMATIC CONTROL}

In order to facilitate trajectory planning and motion control of the 6 DOF robot, control software titled, ' 6 -DOF Robot Motion Control Tool', has been developed for the experimental set-up. This control software provides a userfriendly graphic interface, and was developed with Visual $\mathrm{C}++$ to run in the Microsoft Windows environment.

To perform trajectory planning and motion control, three interfaces; "Trajectory Setting", "Manual Control" (as shown in Fig. 5), and "Motion Control" have been developed. According to whether the user knows the desired trajectory of the robot end-effector in advance, there are two ways to execute trajectory planning. (i) If all intermediate poses of the robot on a desired trajectory are known: the user enters the coordinates of these intermediate poses (3 rotational orientations and 3 translation positions) in the correct sequence in the Trajectory Setting interface. Presently, a total of 50 intermediate poses can be defined on the desired trajectory to allow for complex tasks to be executed (ii) If the desired trajectory path is not known: the user may manually position the robot using the Manual Control interface, by controlling each axis of the robot to achieve a specified end effector position. This is done using a joystick, or by using the software window with a sliding bar for approximate position control, or by clicking the " $<+>$ " or " $<$ - $>$ " button for incremental steps. The "Teach" button can then be used to record the pose coordinates. At present, a maximum of 15 poses can be taught. The pose coordinates determined can then be input to the Trajectory Setting interface in the correct sequence. Presently, the trajectory between consecutive poses consists of straight line segments.

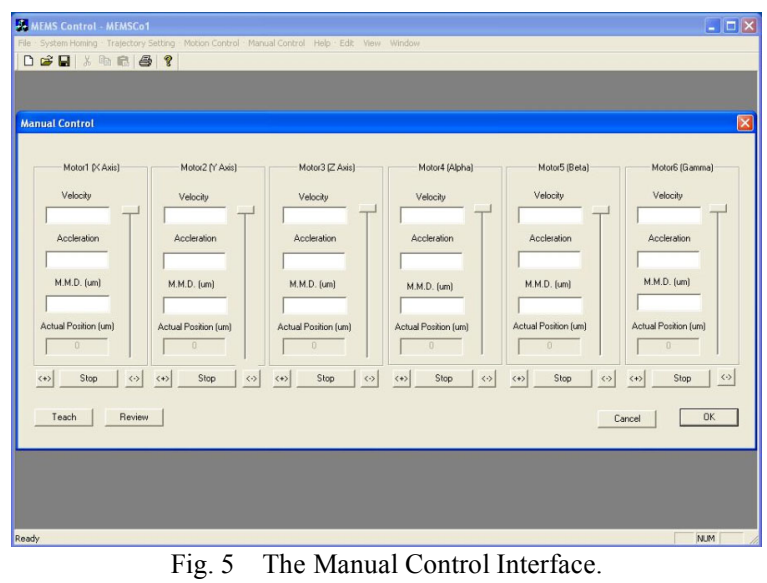


When the desired trajectory is defined, the appropriate velocity and acceleration of each axis are specified in the Motion Control interface. The robot end-effector can then move along the desired trajectory, while the coordinates of each motor are displayed on the interface after the motion is completed. During the programmed motion, at each sample time, the coordinates of each axis will be recorded in a text file to facilitate analysis work. Additionally, collision avoidance software has been incorporated into the system to avoid collisions of the base Cartesian axes with the distal degrees of freedom.

\section{EXPERIMENTAL ASSEMBLY RESULTS}

The 6 DOF robotic manipulator has been successfully used to create 3D MEMS structures. The results presented here are preliminary, but clearly demonstrate the new capabilities of this robot. The microstructures shown in the following examples are not application specific. Rather, they are 'proof of concept' structures that demonstrate possible ways in which micro-parts can be assembled in 5 or 6 DOF. The micro-joints used in these examples are 'key-lock' and 'inter-lock' micromechanical joints [12]. Fig. 6 shows SEM images of a 'Beta Test' microstructure, which demonstrates 5 DOF assembly using the $x, y, z, \alpha$ and $\beta$ axes. Fig. 6(a) shows the first stage of the assembly where the 'lower micro-parts' are assembled at $90^{\circ}$ to the substrate using snap-key-lock joints. Note the insertion 'slits' located on the micro-parts, which will allow for inter-lock joints to be made with other micro-parts at $\beta$ angles of $15^{\circ}, 30^{\circ}, 45^{\circ}, 135^{\circ}, 150^{\circ}$ or $165^{\circ}$ w.r.t. the substrate. Fig. 6(b) shows a completed microstructure that was easily made, with one 'upper micro-part' inserted at $45^{\circ}$ and the other at $135^{\circ}$, w.r.t. the substrate. Note that one micro-part is indeed inserted at $135^{\circ}$ from its original orientation, as its underside is clearly visible (note the dimples). Fig. 7 shows an SEM image of an 'Alpha-Beta Test' structure built with 5 DOF assembly. This structure consists of 'lower micro-parts' joined to a rotational platform that can freely rotate about a fixed hub on the substrate. A set of 'upper micro-parts' are joined at $\beta$-angles of $90^{\circ}$, to create a highly rigid structure. Since the platform may move during assembly, this demonstrates co-ordination between the $\alpha$ and $\beta$ axes. Fig. 8 shows an 'Alpha-Beta-Gamma Test' microstructure, which demonstrates fully 6 DOF assembly using all axes. Fig. 8(a) shows the first stage of assembly where the "lower microparts' are assembled at $90^{\circ}$ to the substrate using snap-keylock joints. Note the different insertion 'slits' located on each of the micro-parts. When used in combination with each other, they allow for inter-lock joints to be made with other micro-parts at an angle of $\beta=45^{\circ}$ and $\gamma=30^{\circ}$, w.r.t. the substrate. Fig. 8(b) shows a partially completed microstructure with the 'upper micro-part' inserted at $\beta=45^{\circ}$ and $\gamma=30^{\circ}$, w.r.t. the substrate. Due to the highly $3 \mathrm{D}$ nature of the microstructure, and the limited depth of focus of the microscope, this micro-joint was very difficult to make since the dual insertion entry-points were not simultaneously visible. To attempt to remedy this problem, a dual microscope system is being considered for future video imaging work.

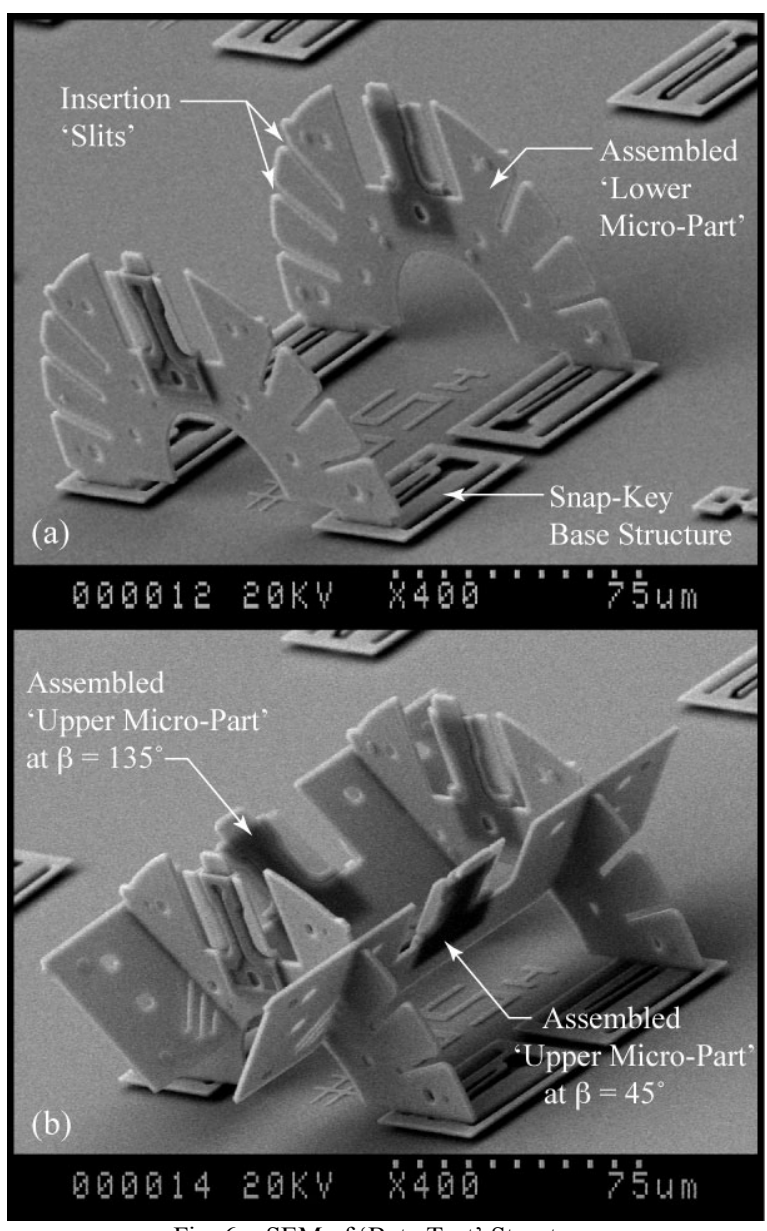

Fig. 6 SEM of 'Beta Test' Structure.

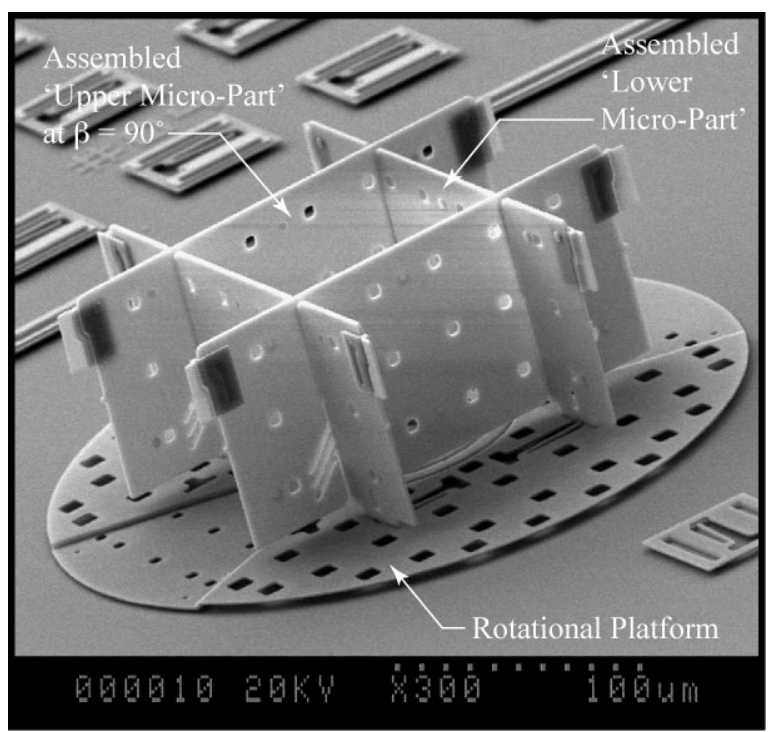

Fig. 7 SEM of 'Alpha-Beta Test' Structure.

The preliminary results of the 5 and 6 DOF assembly system are good. Thus far, 41 'lower micro-parts' of various designs have been successfully joined to double-snap-key-lock joints out of a total of 50 attempts ( $82 \%$ success). Also, 12 'upper micro-parts' of various designs have been successfully inter-lock joined, out of 17 attempts ( $71 \%$ success). 


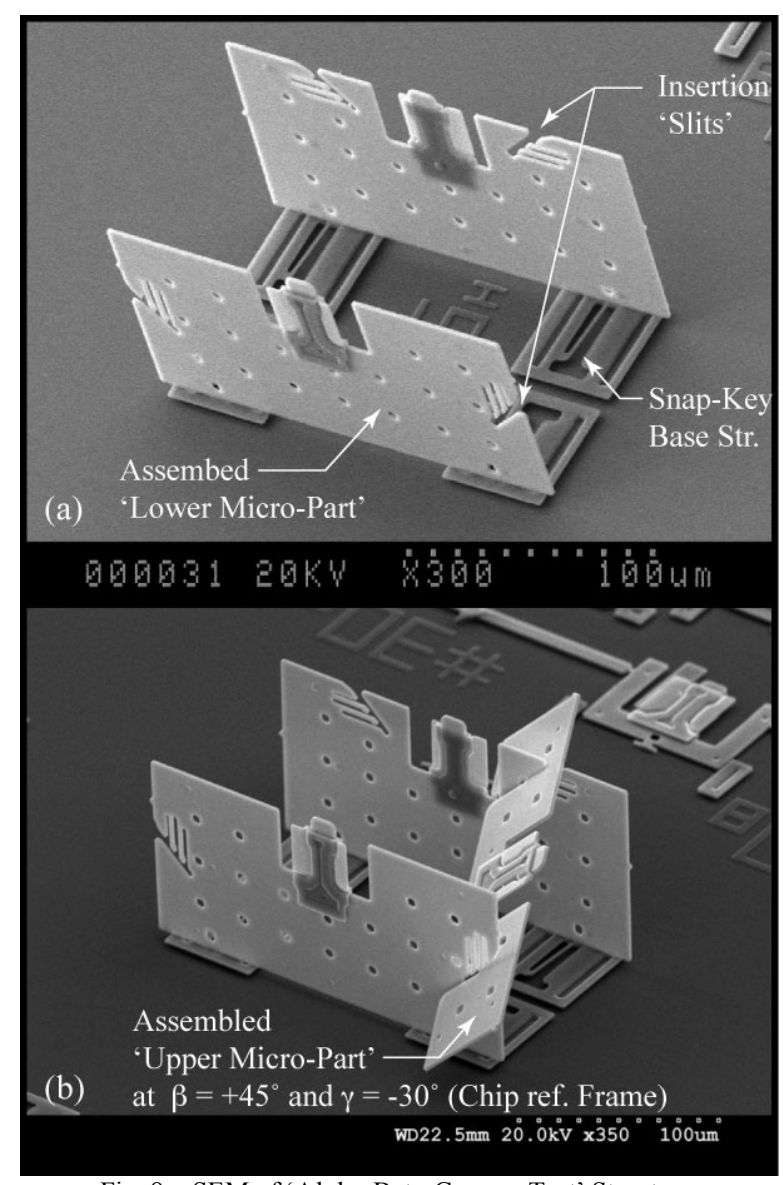

Fig. 8 SEM of 'Alpha-Beta-Gamma Test' Structure.

\section{CONCLUSION}

A new 6 DOF robotic micromanipulator has been developed for the assembly of various micro-parts into 3D MEMS. The robotic manipulator is able to concurrently manipulate all six axes, within a translational workspace of 20 $\mathrm{mm}$ for the $x, y$ and $z$ axes, and within a rotational workspace of $0^{\circ}<\alpha<360^{\circ}, 0^{\circ}<\beta<180^{\circ}$, and $-55^{\circ}<\gamma<+55^{\circ}$. Furthermore, the end-effector (microgripper) is within full view of the video microscope, which allows the entire workspace to be utilized for microassembly operations. For non-concurrent manipulations, the range of rotation is larger, as listed in Table 1. The robot linkages employ high stiffness designs, and the rotational axes employ precision bearings to provide for an end effector repeatability of $+/-2$ microns. The system employs a UV-curable adhesive bonding system to provide reversible, rapid and reliable bonding to microgrippers. The system is controlled using either telerobotic control or automated control, using a newly developed software interface. Some preliminary results have been presented to demonstrate the way in which the system can assemble micro-parts in up to 6 DOF. The new capabilities of this robotic micromanipulator will allow for the assembly of advanced 3D MEMS microstructures and microsystems, that have not been previously possible to construct.

\section{ACKNOWLEDGEMENTS}

This work is supported under a Natural Sciences and Engineering Research Council of Canada (NSERC) Strategic Grant. The authors also wish to thank the Canadian Microelectronics Corp. for allocating MUMPs chip area.

\section{REFERENCES}

[1] N. Dechev, W. L. Cleghorn, James K. Mills, "Microassembly of 3-D MEMS Structures Utilizing a MEMS Microgripper with a Robotic Manipulator", Proc. of IEEE ICRA 2003, Taipei, Taiwan, Sept 2003.

[2] N. Dechev, W. L. Cleghorn, James K. Mills, "Microassembly of 3D Microstructures Using a Compliant, Passive Microgripper", Journal of Microelectromech. Systems, vol. 13, no. 2, April 2004.

[3] D. Koester, A. Cowen, R. Mahadevan, M. Stonefield, and B. Hardy, "PolyMUMPs Design Handbook Revision 9.0", MEMSCAP, MEMS Business Unit (CRONOS), Research Triangle Park, N.C., USA, 2001.

[4] A. Singh, D. Horsely, M. Cohn, A. Pisano, R. Howe, "Batch Transfer of Microstructures Using Flip-Chip Solder Bonding", Journal of Microelectromech. Systems, vol. 8, no. 1, March 1999.

[5] K. F. Harsh, V. M. Bright, and Y. C. Lee, "Solder Self-Assembly for Three-Dimensional Microelectromechanical Systems", Sensors and Actuators A, vol. 77, 1999

[6] J. Zou, J. Chen, C. Liu, and J. E. Schutt-Ainé, "Plastic Deformation Magnetic Assembly (PDMA) of Out-of-Plane Microstructures: Technology and Application", Journal of Microelectromechanical Systems, vol. 10, no. 2, June 2001.

[7] K. W. C. Lai, A. P. Hui, W. J. Li, "Non-Contact Batch Micro-Assembly by Centrifugal Force", Proc. of IEEE International Conference on Micro Electro Mechanical Systems (MEMS 2002), Las Vegas, NV, Jan 2002.

[8] K. Tsui, A. A. Geisberger, M. Ellis, and G. D. Skidmore, "Micromachined End-effector and Techniques for Directed MEMS Assembly", J. of Micromechanics and Microeng., vol. 4, Jan 2004.

[9] G. Yang, J. A. Gaines, and B. J. Nelson, "A Supervisory Wafer-Level 3D Microassembly System for Hybrid MEMS Fabrications", Journal of Intelligent and Robotic Systems, vol. 37, 2003.

[10] E. Shimada, J. A. Thompson, J. Yan, R. Wood, R. S. Fearing, "Prototyping MilliRobots Using Dextrous Microassembly and Folding", Proc. of ASME IMECE / DSCD, Orlando, USA, Nov 2000.

[11] M. Last, V. Subramaniam, K.S.J. Pister, "Out-of-Plane Motion of Assembled Microstructures using a Single-Mask SOI Process", Int. Conf. Transducers 2005, Seoul, Korea, June 2005.

[12] N. Dechev, W. L. Cleghorn, James K. Mills, "Mechanical Fastener Designs for use in the Microassembly of 3D Microstructures", Proc. of ASME IMECE 2004, Anaheim, Calif., Nov 13-19, 2004.

[13] N. Dechev, W. L. Cleghorn, and James K. Mills, "Construction of 3D MEMS Microstructures Using Robotic Microassembly", Workshop in IEEE/RSJ IROS 2003, Las Vegas, USA, Oct 27-31, 2003.

[14] N. Dechev, W. L. Cleghorn and James K. Mills, "Design of Grasping Interface for Microgrippers and Micro-Parts Used in the Microassembly of MEMS," Proc. of the IEEE Int. Conf. on Image Acquisition, Chinese University of Hong Kong, China, June 27-July 3, 2005. 